1 | 2004

NOVECENTO... E DINTORNI

Dire la guerre?

\title{
La guerre dans le cinéma italien Autour du néoréalisme
}

Oreste Sacchelli

\section{(2) OpenEdition}

\section{Journals}

Édition électronique

URL : http://journals.openedition.org/cei/245

DOI : $10.4000 /$ cei.245

ISSN : 2260-779X

Éditeur

UGA Éditions/Université Grenoble Alpes

Édition imprimée

Date de publication : 15 novembre 2004

Pagination : 203-214

ISBN : 978-2-84310-057-4

ISSN : 1770-9571

Référence électronique

Oreste Sacchelli, « La guerre dans le cinéma italien Autour du néoréalisme », Cahiers d'études italiennes [En ligne], 1 | 2004, mis en ligne le 15 mai 2006, consulté le 26 mars 2021. URL : http:// journals.openedition.org/cei/245 ; DOI : https://doi.org/10.4000/cei.245 


\title{
LA GUERRE DANS LE CINÉMA ITALIEN AUTOUR DU NÉORÉALISME
}

\author{
Oreste Sacchelli \\ Université Nancy 2 \\ DÉTERMINANTS POLITIQUES, ÉCONOMIQUES \\ ET SOCIAUX DES PERSONNAGES ET DES SCÉNARIOS
}

À l'instar du western ou de la comédie musicale, le film de guerre est un genre établi comme tel entre autres par la presse dans les rubriques d'information sur les films en programmation. Toutefois, les frontières du genre ne sont pas toujours évidentes car c'est le contenu qui détermine ici le genre et ce contenu peut se prêter à beaucoup de traitements (comédie, drame...). L'information préliminaire sur les films joue sur le sousentendu et la connivence. Les raccourcis font appel à la connaissance préalable que le spectateur a des ingrédients et des codes du genre: s'il est amateur, on lui promet qu'il les retrouvera s'il se donne la peine d'entrer dans la salle indiquée ${ }^{1}$. Dans le cas précis du «film de guerre», le spectateur amateur du genre est sollicité vers un spectacle qui verra s'opposer des hommes presque toujours en uniforme dans le cadre d'un conflit existant ou ayant existé réellement et dont la réalité sera attestée par les accessoires utilisés (armes, matériels...). De ce fait, le spectateur sait presque toujours à l'avance qui sont les gentils et qui sont les méchants ${ }^{2}$. L'intérêt du film

1. Le même type d'information se retrouve dans les programmes des chaînes de télévision ou sur les jaquettes des cassettes vidéo ou des DVD, qui ne sont que les formes plus récentes de consommation cinématographique.

2. Presque: car contrairement aux séries télévisées qui reproduisent systématiquement les mêmes schémas narratifs et les mêmes typologies de personnages, un genre cinématographique fonctionne en renouvelant une partie de ses codes d'un film à l'autre. Ainsi, le manichéisme de base est modulé par l'introduction de nouveaux types de personnages: un ennemi humain, un officier félon, un soldat lâche... 


\section{Oreste SACCHELli}

va donc résider dans la façon qu'aura le scénario de déployer les modalités selon lesquelles l'un ou l'autre camp parviendra à la victoire. À moins que ce schéma simple ne soit débordé par un propos qui utilise le genre pour le transcender et pointer des aspects problématiques contenus dans ses fondements et/ou sa structuration.

Le questionnement sur la détermination des camps paraît donc relativement superflu, contrairement à celui sur les modalités de l'issue du conflit. Toutefois, ne considérer le film de guerre que par le seul biais de la narratologie me paraît réducteur, car il amène à laisser de côté la spécificité du récit cinématographique par rapport à d'autres formes de récit, et tout particulièrement au récit littéraire, ainsi que la spécificité du film dans le champ plus vaste de la communication sociale ${ }^{3}$.

Pour cerner le sens de ce qui nous est raconté dans un film de guerre, un autre type de questionnement me parait plus fécond: il vise à mettre en évidence le point de vue de celui qui raconte ainsi que les intérêts en jeu dans cette communication.

Considérons une séquence absolument topique d'un film de guerre "version aviation»: la mission de bombardement au début du film $U n$ pilota ritorna de Roberto Rossellini, 1942.
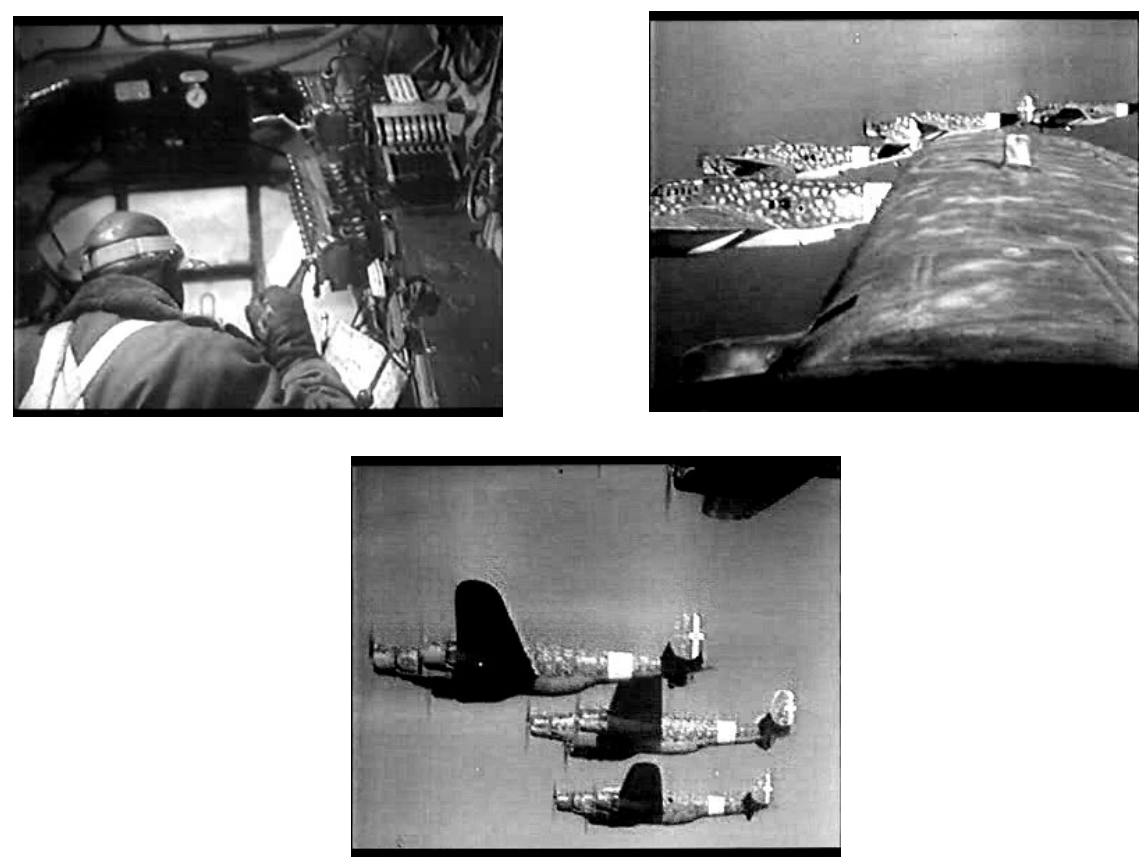

3. Ce qui est particulièrement vrai pour le film de guerre pour les motifs qui seront analysés ci-dessous vaut naturellement pour tout type de film. 
Cette séquence de 6 minutes environ comprend les cinq phases canoniques: le décollage, le parcours vers l'objectif, le lâcher des bombes, le retour, l'atterrissage. Il n'y manque pas non plus les traditionnels nuages de fumée des tirs de la DCA ennemie. La centaine de plans, brefs, donnant lieu à un montage rapide, alternent les vues extérieures (plans larges de l'escadrille ou détails d'un bombardier - vues aériennes du sol, objectif et côte italienne) et les intérieurs de l'avion où se trouve le protagoniste (détail des appareils de navigation, manœuvres diverses et de bombardement, brefs échanges entre les hommes).

Une séquence de ce genre amène deux questions:

- cette séquence est-elle utile?

- quel est son coût?

Ce sont d'ailleurs les deux questions fondamentales auxquelles toute instance de production d'un film (le producteur, avec le réalisateur, éventuellement) doit répondre sauf à risquer les dépassements de budget et les dangers que cela entraîne ${ }^{4}$. Poser ces deux questions ne signifie en aucun cas refuser au film le statut d'œuvre d'art, mais bien au contraire en dégager la spécificité. "Au demeurant, le cinéma est une industrie», disait Malraux, marquant par là la nature composite du cinéma ${ }^{5}$. Rappeler la donnée économique inhérente à la création cinématographique - comme à toute création, sauf divine - ne fait injure à aucun créateur.

À la première question, on ne peut répondre que par l'affirmative. Montrer $\mathrm{du}$ "jamais vu» et amener les spectateurs là où ils ne peuvent pas aller est le fondement même du spectacle cinématographique. La surenchère dans ce domaine a d'ailleurs été le moteur du développement du cinéma, qui tient en cela du spectacle forain, parfois même du manège ${ }^{6}$. Dès l'origine, des équipes de cinéastes furent envoyées dans des contrées lointaines pour ramener des images exotiques, pendant que d'autres exploraient les possibilités du nouvel outil pour déborder le réel par les premiers effets spéciaux. Des films qui donnent des points de vue inédits

4. Les difficultés de la maison de production Titanus suite aux débordements du tournage de $I l$ Gattopardo. Par cet exemple, je n'entends pas dire que certaines scènes coûteuses du film n'auraient pas dû être tournées ou qu'elles sont superflues, mais qu'il aurait mieux valu pour tout le monde, Visconti comme Lombardo, que leur coût fût intégré dans le budget prévisionnel.

5. Le concept de «composition» me paraît plus adapté que celui de «double» dans la mesure où il indique l'aboutissement d'un processus dans une forme qui ne renie pas ses composantes mais considère comme achevés les conflits qui ont pu émailler son histoire.

6. Par le discours de bonimenteur qui promet toujours les choses les plus extraordinaires à l'entrée d'un chapiteau - créatures monstrueuses, animaux exotiques... - ou des sensations de plus en plus fortes de chute, de vitesse... tout à fait semblables à celles que l'on éprouve dans des salles qui mettent le spectateur dans un dispositif différent de celui de la salle de cinéma traditionnelle, comme la Géode à la Cité des Sciences à la Villette ou le Futuroscope à Poitiers. 
sur le monde répondent à une attente du public, comme l'atteste le succès commercial récent de documentaires tels que Microcosmos (Claude Nuridsany, Marie Perennou, 1996) ou Le Peuple migrateur (Jacques Perrin, 2001). Le film de guerre satisfait la pulsion voyeuriste de son spectateur en lui proposant des points de vue que son expérience du monde ne lui offre généralement pas (fort heureusement!): se trouver au milieu d'une bataille ou dans un sous-marin ou dans d'autres situations que la guerre comporte, plus globalement faire l'expérience de la peur, de la violence, de l'horreur et de la mort.

Mais une séquence de ce type a un coût très élevé à cause des matériels à mettre en scène (plusieurs bombardiers) et qui ne sont pas des accessoires faciles à louer.

Dans un ouvrage récent, Hollywood, le Pentagone et Washington, les trois acteurs d'une stratégie globale, Jean-Michel Valentin a montré comment les films de guerre américains ont toujours bénéficié du concours de l'armée, de la marine ou de l'aviation, qui utilisaient la diffusion cinématographique pour promouvoir leur image lorsqu'approchait la date des négociations de leurs budgets au Congrès. Cette coopération vise donc à conjuguer les intérêts du producteur (toujours plus d'images spectaculaires et au moindre coût) et de l'armée qui peaufine son image positive. Cela induit une certaine typologie des personnages ainsi que la mise en avant de l'organisation militaire et de son efficacité, c'est-à-dire le sens général de la trame narrative: le pays - le monde - est menacé par l'ennemi de service (tour à tour les Japonais, les communistes, les extraterrestres ou les terroristes) mais l'armée entre en jeu et annule la menace. Le travail des cinéastes va donc consister à attirer la sympathie du public le plus large possible vers le patriotisme et les personnages qui l'incarnent grâce à l'emploi de vedettes populaires, des solutions narratives et dramaturgiques adaptées, des images spectaculaires et l'introduction d'un suspens bien dosé. Et tout le monde y trouve son compte ${ }^{7}$. Valantin montre comment des films tels qu'Independence day (Roland Emmerich, 1996) ou The final countdown (Nimitz, retour vers l'enfer, Don Taylor, 1980) sont en fait de véritables entreprises de communication rendues indispen-

7. Même les milieux d'affaires, puisque cette sympathie se propage au niveau mondial et entraîne l'exportation de modes de vie et de consommation : films before business. Dans ce sens, la grande consommation de films américains avant les restrictions à l'importation de la fin des années 30 contribue sans doute à la sympathie et à l'admiration des Italiens pour leur ennemi américain, accueilli en libérateur et non en occupant en 1943. Les Anglais, par exemple, ne jouirent pas du même préjugé favorable. Il serait intéressant de connaître la pénétration des productions hollywoodiennes en Afghanistan ou en Irak avant les conflits récents. 
sables par le jeu des divers pouvoirs dans le cadre de la démocratie américaine, fondée sur leur séparation. Un autre élément vient donc s'ajouter à la détermination économique, celui du cadre politique et législatif de l'état dans lequel le projet s'élabore.

Dans l'Italie d'avant-guerre, à partir des mesures prises par le gouvernement fasciste dans le domaine du cinéma au milieu des années $30^{8}$, la situation est plus simple mais l'entreprise de communication est analogue. En Italie aussi, le cinéma est une projection de l'idée nationale, chaque film étant la projection d'une «existence individuelle dans la trame d'un récit collectif " pour reprendre la formule d'Étienne Balibar, cité par JeanMichel Frodon dans son ouvrage La projection nationale - Cinéma et nation. Concernant l'Italie, à laquelle il ne consacre que quelques pages trop rapides (et des assertions discutables), l'auteur écrit:

Le Duce - qui s'est fait installer deux salles de projection pour son usage personnel dans son palais de la Villa Torlonia - commandite lui-même de grandes productions historiques qui exaltent du même élan les origines antiques et les ambitions impérialistes du pouvoir fasciste (Scipion l'Africain de Carmine Gallone, 1937, ou Luciano Serra pilota, 1938, film de guerre contemporain produit par le propre fils du dictateur, Vittorio Mussolini). Les films de ce genre restent néanmoins peu nombreux et, selon un processus déjà observé dans l'Allemagne nazie, ce sont des films de distraction sans ambition particulière (notamment, en Italie, ces comédies futiles qu'on surnomme 'à téléphones blancs') qui bénéficient de l'essentiel du volontarisme politique, économique et législatif clairement manifesté par le pouvoir. (p. 134).

Examinons justement le cas typique d'un film d'aventures tel que Luciano Serra pilota qui paraît sur les écrans en 1938. Il s'agit d'un classique film d'aventures avec happy end.

1921. Luciano Serra est un pilote de la grande guerre qui vit pour sa passion de l'aviation et il la transmet à son fils Aldo. Mais il est réduit à promener de rares touristes fortunés au-dessus du Lac Majeur dans un hydravion, activité peu lucrative qui ne lui permet pas de joindre les deux bouts. Son beau-père, un industriel, lui propose un emploi dans sa société ("Lei deve atterrare!»), il le refuse. Sa femme le quitte emmenant leur fils.

On offre à Luciano un emploi de pilote en Amérique. Il part.

1931. Luciano est au Brésil et il a acquis une certaine renommée. Un journal lui propose de sponsoriser une tentative de vol transatlantique Rio - Rome. Il accepte et l'écho de cette entreprise parvient jusqu'à sa famille. Son fils Aldo part s'engager dans l'aviation.

Luciano décolle de Rio mais il est pris dans une tempête et disparaît en mer.

Aldo est devenu un excellent pilote. Son escadrille est envoyée en Afrique orientale pour contribuer à la pacification d'une région où des pillards sévissent.

Luciano, qui a survécu, s'est engagé sous un faux nom dans la légion. Il est aussi en Afrique orien-

8. Tout commence par la création d'une Direction générale pour la cinématographie au sein du soussecrétariat d'État pour la presse et la propagande (1934), où Luigi Freddi inspire et guide le secteur privé; suit la création de l'Ente Nazionale Industrie Cinematografiche sous l'égide de l'IRI, qui gère des salles, distribue des films - italiens et étrangers - avant de contrôler, par décret, toute l'importation de films étrangers à partir de 1938. Enfin l'Enic est aussi producteur, par participation dans des sociétés ou des consortiums. 


\section{ORESTE SACCHELLI}

tale, dans un train qui est attaqué par les pillards. Aldo, en mission de reconnaissance, vient au secours des assaillis, mais il est blessé et il doit se poser. Son mitrailleur atteint le train et prévient de la présence de l'avion posé tout près avec son pilote blessé. Malgré une blessure, Luciano parvient à atteindre l'avion, à décoller, à gagner la base et à donner l'alerte. Aldo est hors de danger mais Luciano succombe à sa blessure.

Les avions décollent et atteignent rapidement le lieu de la bataille. Ils bombardent les assaillants qui sont vite mis en fuite.

Aldo est rétabli. Une cérémonie est organisée en honneur de son père, décoré à titre posthume.

Les matériaux iconiques proviennent des représentations de la guerre d'Éthiopie véhiculées par les Cinegiornali Luce (images des pillards, mission d'ordre et de civilisation, puissance technologique...) et ils sont insérés dans le scénario classique fondé sur une embuscade montée par un ennemi sournois qui est finalement mis en déroute par l'arrivée des renforts, le classique "arrivano i nostri» qui a servi dans de nombreux westerns, Stagecoach ${ }^{9}$, par exemple. Toutefois, cette trame classique est insérée dans un scénario plus vaste qui correspond au récit national dans lequel Mussolini insère la nation italienne. Comme le démontre fort pertinemment Jean-Michel Frodon, il existe des liens profonds, ontologiques, entre nation et cinéma. Comme le cinéma, la nation est un récit, une fiction, qu'une collectivité se forge et dans lequel elle se projette. Comme la nation, le cinéma est constitué de récits d'existences individuelles inscrites dans la trame d'un récit collectif: ce qu'illustre bien le propos du Duce, lorsque, dans son discours du 9 mai 1936, il montre comment l'empire est un rêve collectif (un récit potentiel) que le fascisme réalise:

Tutti i nodi furono tagliati dalla nostra spada lucente e la vittoria africana resta nella storia della patria, integra e pura, come i legionari caduti e superstiti la sognavano e la volevano. L'Italia ha finalmente il suo impero. Impero fascista, perché porta i segni indistruttibili della volontà e della potenza del Littorio romano, perché questa è la meta verso la quale durante quattordici anni furono sollecitate le energie prorompenti e disciplinate delle giovani, gagliarde generazioni italiane ${ }^{10}$.

Ce récit potentiel inclut la première partie du film et le personnage du héros de la Grande Guerre qui ne trouve pas la place qui lui reviendrait de droit dans la société de l'après-guerre. Humilié, il trouve dans la guerre d'Éthiopie une revanche à sa défaite existentielle. Son sacrifice marque la transmission à son fils des valeurs de la dignité et de l'héroïsme. C'est une parfaite mise en fiction de l'histoire italienne officielle depuis la "vittoria mutilata" jusqu'à la conquête de l'empire. Pour rappeler ce récit collectif à un peuple auquel on demande des sacrifices à cause des effets - réels ou prétendus tels - des sanctions économiques, le régime, représenté par la

9. Film de John Ford, 1939. En français: La Chevauchée fantastique.

10. Relevé sur romacivica. net, le 22 avril 2004. 
Gioventì Italiana del Littorio (GIL), mobilise l'armée dans ce qu'elle a de plus efficace et de plus prestigieusement moderne, l'aviation; le cinéma, en confiant le rôle de Luciano Serra à Amedeo Nazzari, la star masculine la plus populaire du moment, et la réalisation à Goffredo Alessandrini, un cinéaste dont la célébrité égale l'attachement au fascisme; et le capital privé, comme le montre Jean Gili dans L'Italie de Mussolini et son cinéma:

Franco Riganti, directeur de production de Luciano Serra pilota, donne sur la genèse du film des informations extrêmement précises et intéressantes. Selon Riganti, le projet serait né à l'initiative d'un groupe d'industriels milanais. Angelo Monti, gros propriétaire immobilier et industriel du café, était secrétaire d'une association, l'ANIMA (Associazione Nazionale Industriale Motori e Aerei), dans laquelle s'étaient regroupés tous les gros constructeurs d'avions d'Italie: Caproni, Macchi, les chantiers Monfalconi, Savoia Marchetti. Cette association présidée par Gianferrari se propose de financer un film dont devait s'occuper Vittorio Mussolini; Monti souhaite que, pour épauler Vittorio Mussolini, Riganti assume les fonctions de directeur de production. Riganti va à Milan et il reçoit officiellement la charge de l'ANIMA de suivre la préparation et le tournage du film: «Nous n'avons aucune intention de faire du cinéma, déclare Monti, mais il nous a été demandé de financer un film et nous avons affecté un million à ce projet.» (p. 115)

L'aviation est de fait (et va devenir de plus en plus) la grande protagoniste des conflits dans pratiquement tous les théâtres des opérations. La mission de bombardement avec ses phases successives et consécutives est le scénario typique du camp vainqueur. En effet, le vainqueur se distingue du vaincu par la maitrise parfaite de l'enchaînement des causes et des effets qui aboutit à la réalisation du programme qu'il s'était fixé. Les imprévus ne servent qu'à maintenir un minimum de suspens et ils sont assimilés, digérés, dans le déroulement général du programme. Le vaincu, en revanche, voit son propre programme brutalement interrompu par l'irruption d'un imprévu catastrophique et irrécupérable. Une bombe, par exemple. Ebahi, au milieu des décombres (réelles et narratives) il ne peut que constater sa défaite. Le bombardement, selon le point de vue, induit donc deux scénarios opposés. Le néo-réalisme naît de ce changement de point de vue.

La guerre, surtout à partir de 1943, prend l'aspect d'un phénomène incompréhensible parce que nouveau. Le passage du front sur toute la péninsule, précédé par les bombardements aveugles et les destructions, est perçu comme un cataclysme indéchiffrable, parfois comme un châtiment divin. C'est ainsi que dans Roma città aperta Roberto Rossellini (1945) met dans la bouche de Pina des accents dantesques lorsqu'elle demande à Don Pietro: "Ma Cristo non ce vede?». Et la réponse de Don Pietro assimile la guerre au déluge biblique:

Tanti mi fanno questa domanda, Sora Pina. Ma Cristo non ci vede? Ma siamo sicuri di non averlo meritato questo flagello? Siamo sicuri di aver vissuto secondo le leggi del Signore?. 


\section{Oreste SACCHELli}

L'expérience de la première guerre mondiale, dont le théâtre était nettement plus limité, n'apporte pas d'éléments probants pour éclairer la seconde. Dans le film de Luigi Zampa, Vivere in pace (1946), un village de campagne, dans la zone encore occupée par les Allemands, est menacé de destruction car l'un des habitants, Tigna, a hébergé des prisonniers américains évadés. L'un d'eux, Ronald, s'excuse:

- Mi dispiace quello che sta succedendo, Tigna.

- Che ci vuoi fare, Ronald? È colpa de sta guerra maledetta. Almeno prima se la sbrigavano

l'omini. Invece adesso ce vanno de mezzo li ragazzini, le donne, le case.

Cet aspect de la guerre comme fléau incompréhensible est récupéré très vite par la comédie qui se charge ainsi d'échos "qualunquisti» avant la lettre. C'est le cas de Come persi la guerra, de Carlo Borghesio (1945), avec Macario comme interprète principal. Avant guerre, il avait créé un personnage lunaire, un sorte de naïf protégé des dieux qui se sort de situations qui le dépassent sans avoir bien compris ce qui était arrivé. Ce personnage d' «innocent» se glisse parfaitement dans le scénario «réaliste» de la guerre et il semble correspondre parfaitement aux humeurs nationales, vu le succès commercial du film. Dans le final, la guerre étant terminée, Leo ${ }^{11}$ interroge son ami: "Noi, questa guerra, l'abbiamo persa o l'abbiamo vinta?" Tout aussi perplexe, l'ami joue la réponse à pile ou face: "Persa». Leo, qui a ramené toute une série de casques de ses diverses campagnes et des armées auxquelles il a été rattaché après le 8 septembre, les distribue à un groupe d'enfants en même temps qu'il leur distille la morale qu'il a tirée de son expérience de la guerre:

- Adesso mettetevi in fila e giochiamo ai soldati.

- E chi vince?

- Eh, vincono tutti, una volta per uno, tanto non conta niente.

L'innocence (comme fondement psychologique) de Leo dans Come persi la guerra prend une toute autre dimension dans les films que l'on regroupe sous la catégorie générique du «néo-réalisme de guerre ${ }^{12}$ ». Ces films fondent la mythologie de la Résistance et le personnage du partisan, combattant sans uniforme, la mitraillette «Sten» en bandoulière, un foulard (qu'on devine rouge malgré le noir et blanc) autour du cou et, aux pieds, les célèbres «scarpe rotte». Immortalisées par le chant "Fischia il

11. On ne peut s'empêcher de rapprocher ce prénom des «lions» qu'étaient supposés être les Italiens nouveaux forgés par le fascisme.

12. Dans cette catégorie, on trouve les films tournés dans l'immédiat après-guerre et ayant la guerre ou ses conséquences immédiates pour sujet: Giorni di gloria, Mario Serandrei, coréalisé par De Santis, Pagliero, Visconti (1944-1945), Roma città aperta (1945) et Paisà (1946) de Roberto Rossellini, Il sole sorge ancora, d'Aldo Vergano (1946), Caccia tragica, de Giuseppe de Santis (1947), pour l'essentiel. 
vento ${ }^{13}$ ", elles deviennent le symbole de l'esprit de sacrifice qui anime les partisans engagés dans la lutte du peuple italien pour sa rédemption.

C'est justement le thème du sacrifice rédempteur qui permet de lier les deux phases initiales et apparemment contradictoires de l'œuvre de Roberto Rossellini, la trilogie de la guerre fasciste (La nave bianca - 1941, Un pilota ritorna - 1942, L'uomo della croce - 1943) et la trilogie de la guerre antifasciste (Roma città aperta - 1945, Paisà - 1946, Germania anno 0 - 1948) ${ }^{14}$. Toute la dernière partie de L'uomo della croce se déroule pendant une bataille incompréhensible: on ne sait qui des Russes ou des Italiens tient le terrain, ni d'où viennent les obus qui tombent à côté de la maison en ruine où se sont abrités des blessés des deux camps, des femmes russes et l'aumônier militaire italien qui soutient tout le monde. Il apporte d'ailleurs un grand réconfort aux femmes russes que le bolchevisme n'a pas déchristianisées (l'innocence d'un peuple... déjà) avant de donner sa vie pour assister un mourant.

L'esprit de sacrifice est aussi la caractéristique fondamentale du personnage de Don Pietro dans Roma città aperta. On sait que le projet initial du film concernait seulement un prêtre ayant réellement été fusillé pour faits de résistance (Don Morosini), et que le personnage du communiste Manfredi s'est ajouté suite aux insistances de Sergio Amidei, scénariste du film et militant communiste. Ainsi, c'est fusillé comme son modèle que meurt Don Pietro, après s'être défendu des accusations d'actes nuisant à l'armée allemande d'un simple rappel de sa mission évangélique:

- Come voi chiamate un uomo che non solo fornisce documenti falsi e rifugio a italiani che preparano attentati contro i nostri soldati, ma dà asilo e aiuto persino a disertori tedeschi?

- Un uomo che indegnamente cerca di esercitare la carità.

Son exécution, qui suit la mort de Manfredi, pose les bases du mythe de l'unité entre catholiques et communistes contre le mal absolu représenté par le nazisme et le fascisme. Mais cette scène proclame aussi l'innocence du peuple italien que vingt ans de fascisme n'ont pas contaminé. Les soldats italiens de l'armée de la République de Salò qui doivent exécuter le prêtre ont tous le réflexe de baisser leur arme et tirer au sol. Respect du sacré ou respect du résistant, cet acte de désobéissance prouve une solidarité instinctive avec le nouveau pouvoir (l'Église, la Démocratie chrétienne, le CLN...) qui est en train d'émerger.

13. Fischia il vento e infuria la bufera,/scarpe rotte e pur bisogna andar,/ a conquistare la rossa primavera,/dove sorge il sol dell'avvenir.

14. Sur cette question, on pourra consulter: Enrique Seknadje-Askenazi, Roberto Rossellini et la seconde guerre mondiale - Un cinéaste entre propagande et réalisme, Paris, L'Harmattan, 2000. 


\section{ORESTE SACCHELLi}

Les films du "néo-réalisme de guerre», et ceux de Rossellini tout particulièrement ${ }^{15}$, véhiculent une image nécessaire aux Italiens en vue de la constitution d'une identité nouvelle pour remplacer celle qui s'est perdue dans le désastre de la guerre, pour recommencer un récit national satisfaisant autant pour eux-mêmes que comme nation nouvelle dans le concert des nations de l'après-guerre. Le discours de De Gasperi à la conférence de la paix (10 août 1946), plaidant la cause italienne, s'appuyait sur des idées-forces analogues:

Sento il diritto di parlare [...] come democratico antifascista, come rappresentante della nuova repubblica che, armonizzando in sé le aspirazioni umanitarie di Giuseppe Mazzini, le concezioni universaliste del cristianesimo e le speranze internazionaliste dei lavoratori, è tuttora rivolta verso quella pace duratura e ricostruttiva che voi cercate e verso quella cooperazione fra i popoli che avete il compito di stabilire ${ }^{16}$.

Gian Piero Brunetta ${ }^{17}$ rappelle ce qu'écrivait en décembre 1946 la revue Fotogrammi:

In pochi mesi Rossellini e Coppi hanno fatto bene all'Italia più dei signori De Gasperi e Togliatti.

Dès 1948, après la victoire de la Démocratie chrétienne aux premières élections législatives, l'identité de l'Italie nouvelle semble définitivement établie, du moins de l'avis du camp vainqueur. La reprise en main du cinéma s'opère aussitôt. C'est d'abord la polémique des démocrates chrétiens contre le néoréalisme, suivie par la loi de 1949 qui instaure un système d'aide au cinéma en échange d'un alignement idéologique. La «renaissance» du peuple italien est désormais évoquée à travers de jeunes personnages optimistes que rien n'arrête (surtout pas les difficultés économiques), inscrits dans des scénarios dynamiques où la volonté et la joie de vivre déplacent les montagnes ${ }^{18}$. L'évocation de la guerre et du fascisme disparaît des écrans, avant même que l'examen de conscience ait réellement commencé. Le débat politique lui-même s'est déplacé et concerne désormais la mise en œuvre de la Constitution de la République. Le rappel des idéaux de la Résistance est le cheval de bataille du Parti communiste:

15. L'année suivante, Rossellini réalise Paisà qui raconte en six épisodes la construction de l'union entre peuple italien et forces américaines dans la lutte contre les forces du mal (nazisme et fascisme), une sorte de plaidoyer avant la lettre pour que l'Italie soit admise dans ce que sera l'Otan.

16. Cité dans Silvio Lanaro, Storia dell'Italia repubblicana, Venezia, Marsilio, 1992, p.18-19.

17. Gian Piero Brunetta, Identità italiana e identità europea nel cinema italiano, Torino, Edizioni della Fondazione Gianni Agnelli, 1996, p. 16.

18. Due soldi di speranza, de Renato Castellani (1951), inaugure le "néoréalisme rose». Les recettes record de ce film, confrontées à l'échec commercial de Umberto D (De Sica, 1951), marquent le dépassement du néoréalisme et son adaptation à des formes commerciales. Suivent les séries des Don Camillo et des Pane, amore..., autant de succès commerciaux. 
on ne s'étonnera pas que ce thème ne trouve pas de traduction cinématographique dans le contexte d'intégration capitaliste de l'Italie aux institutions occidentales. Il faut attendre près de dix ans pour que le cinéma italien s'intéresse de nouveau à l'époque du fascisme et de la guerre, et cela se produit dans un contexte économique et idéologique consolidé, le boom économique, qui marque l'aboutissement du processus d'intégration occidentale débuté le 10 juillet 1943, lorsque les premiers soldats américains ont débarqué sur les côtes de Sicile.

\section{Bibliographie}

Frodon Jean-Michel, La projection nationale. Cinéma et nation, Éditions Odile Jacob, Paris, 1998. Valentin Jean-Michel, Hollywood, le Pentagone et Washington. Les trois acteurs d'une stratégie globale, Éditions Autrement, Paris, 2003.

Brunetta Gian Piero, Identità italiana e identità europea nel cinema italiano, Torino, Edizioni della Fondazione Gianni Agnelli, 1996.

Gili Jean A., L'Italie de Mussolini et son cinéma, Paris, Henri Veyrier, 1985.

Seknadje-Askenazi Enrique, Roberto Rossellini et la seconde guerre mondiale. Un cinéaste entre propagande et réalisme, Paris, L'Harmattan, 2000.

Lanaro Silvio, Storia dell'Italia repubblicana, Venezia, Marsilio, 1992.

\section{Films cités}

Luciano Serra pilota, Luigi Alessandrini, Italie, 1938.

Stagecoach, John Ford, USA 1939.

La nave bianca, Roberto Rossellini, Italie, 1941.

Un pilota ritorna, Roberto Rossellini, Italie, 1942.

L'uomo della croce, Roberto Rossellini, Italie, 1943.

Giorni di gloria, Mario Serandrei, coréal. De Santis, Pagliero, Visconti, Italie, 1944-1945.

Come persi la guerra, de Carlo Borghesio, Italie, 1945.

Roma città aperta, Roberto Rossellini, Italie, 1945.

Il sole sorge ancora, Aldo Vergano, Italie, 1946.

Paisà, Roberto Rossellini, Italie, 1946.

Vivere in pace, Luigi Zampa, Italie, 1946.

Caccia tragica, Giuseppe de Santis, Italie, 1947.

Germania anno 0, Roberto Rossellini, Italie, 1948.

Due soldi di speranza, de Renato Castellani, Italie, 1951.

Umberto D., Vittorio De Sica, Italie, 1951.

The final Countdown, Don Taylor, USA, 1980.

Independence day, Roland Emmerich, USA, 1996.

Microcosmos, Claude Nuridsany, Marie Perennou, France, 1996.

Le Peuple migrateur, Jacques Perrin, France, 2001. 
